\title{
Treatment simplification in HIV-infected adults as a strategy to prevent toxicity, improve adherence, quality of life and decrease healthcare costs
}

This article was published in the following Dove Press journal:

Patient Preference and Adherence

18 July 201 I

Number of times this article has been viewed

\author{
Jean B Nachega ${ }^{1-3}$ \\ Michael J Mugavero 4 \\ Michele Zeier ${ }^{2}$ \\ Marco Vitória ${ }^{5}$ \\ Joel E Gallant ${ }^{3,6}$
}

'Department of International Health, Johns Hopkins Bloomberg School of Public Health, Baltimore, MD, USA; ${ }^{2}$ Department of Medicine and Centre for Infectious Diseases (CID), Stellenbosch University, Faculty of Health Sciences, Cape Town, South Africa; ${ }^{3}$ Department of Epidemiology, Johns Hopkins Bloomberg School of Public Health, Baltimore, MD, USA; ${ }^{4}$ Division of Infectious Diseases, Department of Medicine, University of Alabama at Birmingham, Birmingham, AL, USA; ${ }^{5}$ HIV Department, World Health Organization, Geneva, Switzerland; 'Department of Medicine, Division of Infectious Diseases, Johns Hopkins University School of Medicine, Baltimore, MD, USA

\section{Video abstract}

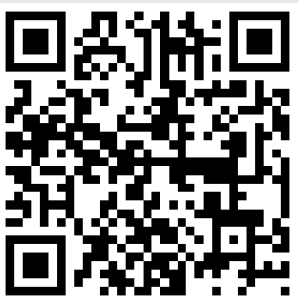

Point your

smartphone at the $\mathrm{QR}$ code to the left. If you have a QR code reader the video abstract will appear. Or use: http://dvpr.es/ nachega

Correspondence: Jean B Nachega Johns Hopkins University, Bloomberg School of Public Health, Department of International Health, Global Disease Epidemiology and Control Program, $6 I 5$ N. Wolfe Street, Suite W503I, Baltimore, Maryland 21205 , USA

$\mathrm{Tel}+\mathrm{I} 4109552378$

Fax + I 4I0 5026733

Email jnachega@jhsph.edu

\begin{abstract}
Since the advent of highly active antiretroviral therapy (HAART), the treatment of human immunodeficiency virus (HIV) infection has become more potent and better tolerated. While the current treatment regimens still have limitations, they are more effective, more convenient, and less toxic than regimens used in the early HAART era, and new agents, formulations and strategies continue to be developed. Simplification of therapy is an option for many patients currently being treated with antiretroviral therapy (ART). The main goals are to reduce pill burden, improve quality of life and enhance medication adherence, while minimizing short- and long-term toxicities, reducing the risk of virologic failure and maximizing cost-effectiveness. ART simplification strategies that are currently used or are under study include the use of once-daily regimens, less toxic drugs, fixed-dose coformulations and induction-maintenance approaches. Improved adherence and persistence have been observed with the adoption of some of these strategies. The role of regimen simplification has implications not only for individual patients, but also for health care policy. With increased interest in ART regimen simplification, it is critical to study not only implications for individual tolerability, toxicity, adherence, persistence and virologic efficacy, but also cost, scalability, and potential for dissemination and implementation, such that limited human and financial resources are optimally allocated for maximal efficiency, coverage and sustainability of global HIV/AIDS treatment.
\end{abstract}

Keywords: ART, simplification, adherence, persistence, once-daily, coformulations, healthcare cost, quality of life

\section{Introduction}

Since the advent of highly active antiretroviral therapy (HAART), the treatment of human immunodeficiency virus (HIV) infection has become more potent and better tolerated. While the current treatment regimens still have limitations, they are more effective, more convenient, and less toxic than regimens used in the early HAART era, and new agents, formulations and strategies continue to be developed. ${ }^{1}$

Simplification of therapy is an option for many patients currently being treated with antiretroviral therapy (ART). The main goals are to reduce pill burden and to improve quality of life and medication adherence, while minimizing short- and longterm toxicities, reducing the risk of virologic failure, preserving future treatment options, maximizing cost-effectiveness and ultimately reducing the occurrence of clinical events and disease progression. ${ }^{1}$

ART simplification strategies that have been used or are under study include the use of fixed-dose combinations, adoption of once-daily regimens with less toxic drugs, and more recent induction-maintenance approaches. ${ }^{1,2}$ Intermittent ART strategies, 
previously felt to be promising approaches for treatment simplification, have now been discredited in clinical trials.

In this article, we review the major aspects of these simplification strategies and discuss their ability to improve ART adherence and persistence, clinical outcomes, toxicity and quality of life as well as the potential impact on healthcare costs.

\section{Methods}

We retrieved published English language studies via PubMed, Medline and Google Scholar through January 2011. Our search terms included "HIV", "treatment simplification"; "adherence"; "HAART"; "ART"; "cART"; "patient preference"; "healthcare cost". Abstracts from major HIV/AIDS or infectious diseases conferences such as Conference on Retrovirus and Opportunistic Infections (CROI), International AIDS Society (IAS), International AIDS Conference, International Conference on Antimicrobials Agents and Chemotherapy (ICAAC) and Infectious Diseases Society of America (IDSA) were also reviewed for inclusion.

\section{Fixed-dose combinations and once-daily regimens Fixed-dose combinations or coformulations}

Fixed-dose combinations or coformulations (FDCs) are products that combine two or more active drugs in one tablet or capsule. There are many potential advantages and disadvantages of FDCs (Table 1). A reduction in pill burden has been associated with improved ART adherence (Figure 1). Possible limitations include the lack of pediatric equivalents, inadequate provision for lead-in dosing and a number of other shortcomings concerning availability, packaging and provision for reporting adverse events (Table 1). As an example, the

Table I Avantages and disadvantages of fixed-doses combinations (FDCs)

\begin{tabular}{l}
\hline Advantages of FDCs \\
Decreased pill burden \\
Better adherence \\
Prescription errors less likely \\
Patients unable to take partial regimen \\
Experience of FDCs with other diseases such as tuberculosis, malaria etc \\
Practical for management in large programs (improved drug supply systems) \\
Cheaper in generic form (eg, in resource-limited settings) \\
Disadvantages of FDCs \\
Does not accommodate lead-in dose \\
Difficult to use when dose adjustments are needed (eg, renal failure) \\
Need to stop FDC for adverse drug reaction to one component \\
Limited availability of pediatric formulations \\
More expensive if generic version of one or more components available \\
(developed countries)
\end{tabular}

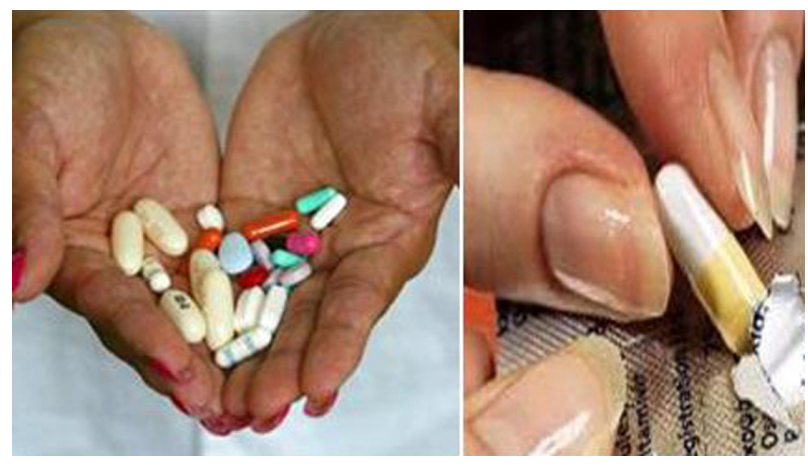

Figure I 1996 to 20 I I, from multiple drugs to once-daily and more potent antiretroviral therapy regimens: the long road to patient satisfaction and adherence.

three-drug, single tablet coformulation of tenofovir disoproxil fumarate (TDF), emtricitabine (FTC) and efavirenz (EFV) is approved for once-daily dosing in the US and is the first available fixed-dose combination tablet containing all components of a preferred antiretroviral regimen (Table 2). Several generic pharmaceutical manufacturers produce low priced FDCs for once- or twice-daily dosing ${ }^{3}$. Questions have been raised about the bioequivalence of such generic formulations, but so far published clinical trial data have been reassuring, and these generic FDCs are being approved by the US FDA for use in President's Emergency Plan for AIDS relief (PEPFAR) programs in resource-limited settings. ${ }^{4,5}$

\section{Once-daily regimens and impact on treatment adherence and persistence}

Parienti and colleagues investigated treatment adherence with once-daily regimens, assessing 11 randomized, controlled

Table 2 Approved and under study antiretroviral therapy coformulations

\begin{tabular}{ll}
\hline & Type \\
\hline Approved coformulations & \\
AZT/3TC & Dual NRTI \\
d4T/3TC & Dual NRTI \\
ABC/3TC* & Dual NRTI \\
TDF/FTC* & Dual NRTI \\
TDF/FTC/EF* & Dual NRTI + NNRTI \\
TDF/3TC/EFV & Dual NRTI + NNRTI \\
AZT/3TC/NVP & Dual NRTI + NNRTI \\
d4T/3TC/NVP & Dual NRTI + NNRTI \\
AZT/3TC/ABC* & Triple NRTI \\
LPV/r* & Boosted PI \\
Coformulations under study & \\
TDF/FTC/rilpivirine & Dual NRTI + NNRTI \\
TDF/FTC/elvitegravir/cobicistat & Dual NRTI + boosted integrase inhibitor \\
\hline Note: *Avatin Units
\end{tabular}

Note: *Available in United States.

Abbreviations: AZT, zidovudine; $3 \mathrm{TC}$, lamivudine; $\mathrm{d} 4 \mathrm{~T}$, stavudine; $\mathrm{ABC}$, abacavir; $T D F$, tenofovir disoproxil fumarate; FTC, emtricitabine; EFV, efavirenz; LPV/r, lopinavir/ritonavir; NRTI, nucleoside reverse transcriptase inhibitor; NNRTI, nonnucleoside reverse transcriptase inhibitor; Pl, protease inhibitor. 
trials with a total of 3029 subjects. ${ }^{6}$ In this meta-analysis, adherence rates were modestly better with once-daily regimens $(+2.9 \%$; $95 \%$ confidence interval $(\mathrm{CI}), 1.0 \%-4.8 \%$; $P<0.003)$ than with twice-daily regimens. The effect size was more pronounced for ART-naïve patients and when all medications were taken once daily. Of note, 48-week virologic suppression with once- and twice-daily regimens was similar ( $77 \%$ versus $76 \%$, respectively). More recent studies comparing once-daily regimens to more frequently dosed regimens continue to support the positive impact of once-daily regimens on ART adherence (Table 3). Maitland and colleagues performed a randomized trial in which patients were switched from abacavir (ABC) plus lamivudine (3TC) BID to a $\mathrm{QD}$ fixed-dose formulation of $\mathrm{ABC} / 3 \mathrm{TC}$ and demonstrated that an all-QD regimen improved multiple measures of adherence (via MEMScap) and patient satisfaction (measured by HIV treatment satisfaction questionnaire [HIVSTQ] score) with treatment over 4 and 8 weeks of follow-up. ${ }^{7}$ A randomized controlled trial by Wright and colleagues evaluating safety and antiviral activity of QD versus BID enfuvirtide documented better adherence (95\% versus $80 \%$ ) of prescribed doses with the once-daily strategy. ${ }^{8}$ In another randomized trial, 320 patients with viral load $<50$ copies/mL on ART regimens dosed at least twice-daily were randomized to switch to a once-daily regimen (EFV, extended-release stavudine [d4TxR], and 3TC) or to continue existing therapy. ${ }^{9}$ Adherence was monitored using MEMS caps, an AIDS Clinical Trials Group (ACTG)-validated questionnaire, and pill counts. By the primary efficacy measure (proportion of patients who maintained virologic suppression at Week 48), the once-daily arm was non inferior $(80.0 \%)$ to the twicedaily arm (75.8\%), and $91.0 \%$ of patients preferred the simpler regimen. ${ }^{9}$

Following US FDA approval in 2009 of lopinavir/ ritonavir (LPV/r) tablets for once daily use in treatment-naïve patients, the results of the M06-802 trial became available. ${ }^{10}$ In this study, 599 patients failing ART with a viral load $>1000$ copies/mL were randomized 1:1 to taking LPV/r once daily or twice daily, both arms at a total daily dose of $800 / 200 \mathrm{mg}$. Trial participants had to be naïve to LPV/r and the choice as to the background regimen was made by the treating investigator, who had genotyping testing available. The once-daily arm was found to be non-inferior in terms of efficacy, and toxicity, notably diarrhea, was not more frequent in the once-daily arm. Non-inferiority for efficacy could not be evaluated for patients with 3 or more significant protease inhibitor (PI) mutations, as only 27 such subjects participated. Treatment adherence was significantly better for the once-daily arm. Although pill burden is unchanged by once-daily adminstration of LPV/r, this does provide an opportunity of simplifying regimen in countries with limited choice of PI; in many cases it is the only one offered on treatment programs. The FDA approved once daily administration of LPV/r in treatment experienced persons in 2010 , but does not recommend once daily dosing for adult patients with three or more LPV resistance-associated substitutions: L10F/I/R/V, K20M/N/R, L24I, L33F, M36I, I47V, G48V, $\mathrm{I} 54 \mathrm{~L} / \mathrm{T} / \mathrm{V}, \mathrm{V} 82 \mathrm{~A} / \mathrm{C} / \mathrm{F} / \mathrm{S} / \mathrm{T}$, and I84V. ${ }^{10}$

Once-daily, fixed-dose regimens are also attractive for difficult-to-treat patients, such as injecting drug users and the homeless and other marginally housed HIV-infected individuals. To address this question, the single-tablet regimen of $\mathrm{TDF} / \mathrm{FTC} / \mathrm{EFV}$ was compared to regimens taken more than once daily. ${ }^{11}$ Adherence was significantly higher with the single-tablet regimen $(P=0.006)$, with better viral suppression (HIV RNA < 50 copies/mL: $69.2 \%$ versus $46.5 \%$; $P=0.02$ ) Finally, using a mixed effect model, De Jesus and colleagues found a lower daily pill burden with the single tablet regimen of TDF/FTC/EFV $(\mathrm{n}=157)$ or TDF/FTC + EFV ( 2 tablets/day, $\mathrm{n}=162$ ) compared to TDF + FTC + EFV (3 tablets/day, $\mathrm{n}=238)\left(P=0.0005\right.$ and 0.0262 , respectively). ${ }^{12}$ In developed countries, preferred initial regimens containing the ritonavir-boosted protease inhibitors, atazanavir (ATV/r) and darunavir (DRV/r), represent once-daily options, in addition to once-daily non-nucleoside reverse transcriptase inhibitor (NNRTI)-based regimens. ${ }^{1}$

Reduction in pill burden may also improve adherence and outcome, even with regimens that are already dosed once-daily. The recent ADONE (ADherence to ONE pill) study, a prospective, multicenter study in which 212 patients on suppressive once-daily regimens containing TDF, EFV plus either FTC or 3TC, were switched to the single-tablet regimen of FTC/TDF/EFV ${ }^{13}$ Reducing the pill burden from three or two pills to one pill per day resulted in improved adherence (to $96.1 \%$ from $93.8 \% ; P<0.01$ ), which continued through 6 months of treatment (96.2\%). Quality of life improved from $68.8 \%$ to $72.7 \%(P=0.042)$.

Dosing frequency and pill burden have also been identified as important treatment characteristics for treatment persistence. ${ }^{14}$ Distinct from but related to medication adherence, persistence reflects the duration of time from initiation to discontinuation of therapy, and can be measured at the regimen or patient level. In developed countries, improved regimen persistence, or durability, has been observed with regimens dosed once-daily and containing fewer pills. ${ }^{15}$ In general, NNRTI-based regimens (versus PI- and ritonavir 


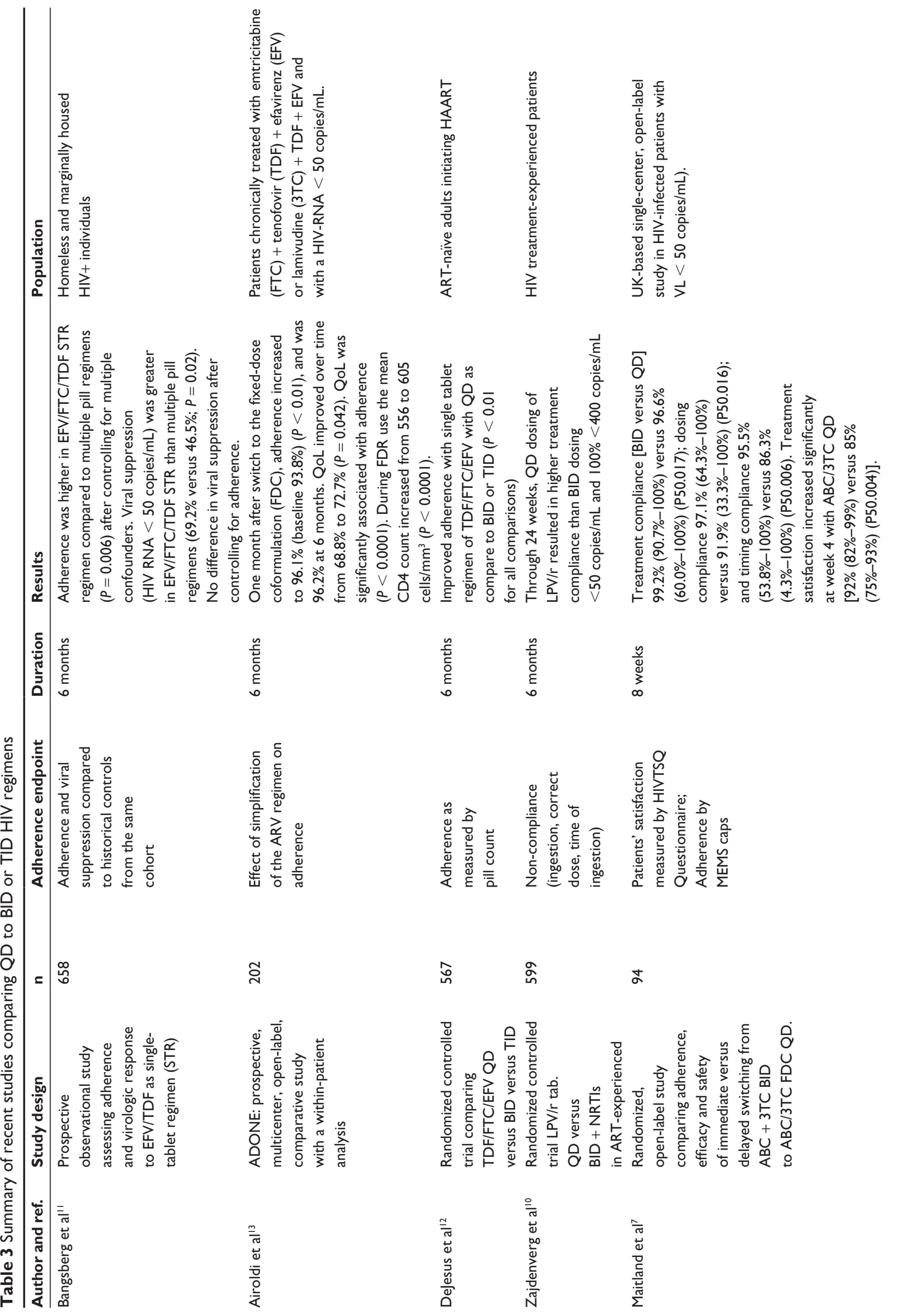




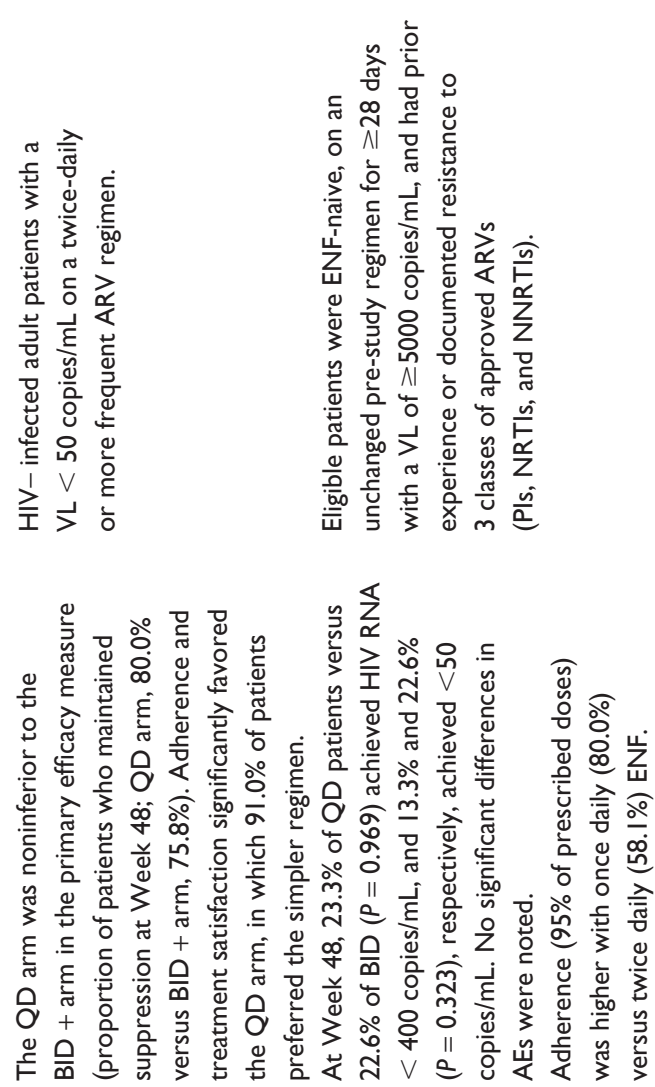

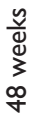
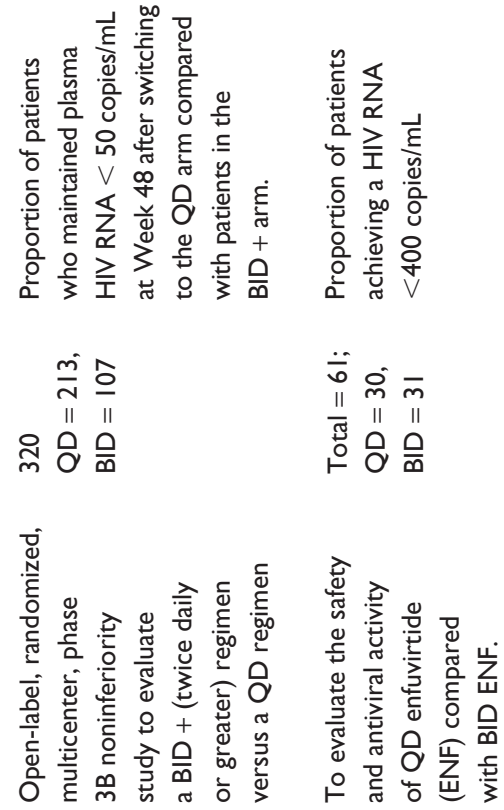

$\frac{0}{0}$
$\stackrel{0}{0}$
$\frac{0}{\widehat{ก}}$

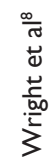

[RTV] boosted PI [PI/r]-based regimens), and newer nonthymidine analog NRTIs TDF and ABC have demonstrated improved regimen persistence when compared with boosted or unboosted PIs, thymidine nucleoside NRTIs (ZDV and $\mathrm{d} 4 \mathrm{~T}$ ) and didanosine (ddI). This is likely to be due both to the availability of these agents as components of fixed-dose combinations dosed once-daily, and to their improved tolerability and toxicity profiles. ${ }^{16}$ Not surprisingly, the use of FDCs has also been associated with a lower frequency of differential adherence among their constituent medications compared with regimen components administered separately. ${ }^{16}$ Among PIs, the once-daily boosted-PIs ATV/r and DRV/r have shown improved persistence attributable to fewer toxicity driven regimen switches with these agents compare to other RTVboosted PI regimens. ${ }^{17}$

\section{Intermittent antiretroviral therapy}

There was hope that intermittent antiretroviral therapy might decrease side effects and toxicity while maintaining virologic suppression. Intermittent strategies have included interruption and re-initiation of ART determined by either pre-specified CD4 thresholds or time intervals. Early pilot studies of short-cycle structured treatment interruption (STI) used cycles of 7 days on therapy followed by 7 days off. In the first pilot study, 10 participants were given d4T, 3TC, and ritonavir-boosted indinavir (IDV/r) for up to 68 weeks. ${ }^{18}$ While viral rebound ( $>50$ copies $/ \mathrm{mL}$ ) occurred during the off-treatment periods, no resistance was detected, there were no changes in CD4 cell counts, and lipids levels declined significantly. In a subsequent study of 7-day on/off cycles of therapy with an EFV-based regimen, 7 of 8 participants maintained virologic suppression for $60-84$ weeks. ${ }^{19}$ The Five on, Two Off (FOTO) study used a shorter cycle of 5 days on and 2 days off therapy. ${ }^{20,21}$ Thirty subjects were enrolled, 10 on EFV-based regimens, 10 on NVP-based regimens and 10 on PI-based regimens. At 24 weeks, as-treated analysis showed that virologic suppression was maintained in 26 of 29 subjects $(89.6 \%)$. None of the EFV-treated subjects developed virologic failure, which was attributed to the long half-life of EFV. In addition, patients maintained virologic suppression through week 48. No virologic failures were noted in either arm.

Three clinical trials (Trivacan, Staccato, and SMART) evaluated the safety and efficacy of intermittent therapy using a CD4-guided approach. The French-funded Trivacan ANRS 1269 trial enrolled 386 HIV-infected adults from Côte d'Ivoire. ${ }^{22}$ Participants were randomized to receive continuous therapy (CT) or CD4-guided therapy as the intermittent 
regimen (IT), with therapy interruption and re-initiation thresholds of 350 and 250 cells $/ \mathrm{mm}^{3}$, respectively. Primary endpoints were severe AIDS-related morbidity and death. The incidence of severe morbidity per 100 person-years was higher in the IT group (17.6) compared to the CT group (6.7; $P<0.01$ ), with the most frequent event being invasive bacterial infections. This difference led to premature termination of the study. There was no statistically significant difference in mortality. In the Staccato Trial, CD4-guided treatment interruptions were evaluated in participants from Thailand, Switzerland and Australia. ${ }^{23}$ Four hundred and thirty participants with CD4 counts greater than 350 cells $/ \mathrm{mm}^{3}$ and HIV RNA $<50$ copies $/ \mathrm{mL}$ were randomized to continued antiretroviral therapy (CT) or scheduled IT. Therapy was restarted after a confirmed CD4 count below 350 cells $/ \mathrm{mm}^{3}$ in the IT arm. The primary study endpoints were the proportion of participants with HIV RNA $<50$ copies $/ \mathrm{mL}$ at the end of the trial and cumulative ART use in both groups. Reduced medication use in the IT group, indicated as a "drug savings" compared to the CT group of $61.5 \%$, was reported. HIV RNA levels were below 50 copies $/ \mathrm{mL}$ in $90.5 \%$ and $91.8 \%$ of the participants in the IT and CT groups, respectively. At the end of trial, the IT group had lower mean CD4 counts compared to the $\mathrm{CT}$ group $(P<0.01)$, and fewer subjects maintained a CD4 cell count above 350 cells $/ \mathrm{mm}^{3}$ in the IT versus the CT group $(P<0.0001)$. The investigators concluded that a substantial drug savings occurred in the IT group, and that treatment-related adverse events were more common in the CT group.

The SMART study enrolled 5472 patients with CD4 counts $>350$ cells $/ \mathrm{mm}^{3}$, randomized to continuous or episodic ART (CT or IT). ${ }^{24-28}$ Participants in the IT group deferred therapy until the CD4 cell count was $<250$ cells $/ \mathrm{mm}^{3}$. After an average follow-up of 16 months, opportunistic infections or death from any cause occurred in 120 IT participants (3.3 events per 100 person-years) compared with 47 in the CT group (1.3 events per 100 person-years), giving a hazard ratio of $2.6(P<0.001)$ for the IT group. The trial was stopped prematurely, and the investigators concluded that intermittent ART increased the risk of opportunistic diseases and death. Of note, an unexpected finding was that the incidence of cardiovascular, renal and hepatic disease was higher in the IT group, whereas it was assumed that ART would increase the risk of such adverse outcomes. More recently, observational data from the Swiss cohort study ${ }^{26}$ showed that interruption of ART limits CD4 cell recovery and increases the risk for opportunistic complications and death. In this study, persons receiving continuous ART experienced greater CD4 cell recovery and a reduced risk for opportunistic complications and death. CD4 cell recovery was less pronounced in persons with treatment interruptions more than 6 months.

Therefore, CD4-guided treatment interruption is not a recommended strategy, based largely on the greater risk of CD4 decline, morbidity, and mortality observed in several clinical studies. Results have been more favorable with shorter interruptions based on pre-specified time intervals, such as the FOTO approach, but this strategy has only been studied in small pilot trials. Based on the available evidence, intermittent therapy strategies are not recommended.

\section{Induction-maintenance strategies}

While earlier studies involving switches from standard therapy to less active maintenance regimens failed, ${ }^{29,30}$ studies involving maintenance with triple-NRTI therapy (ZDV/3TC/ $\mathrm{ABC}$ ) following induction with standard regimens demonstrated some promise, although with inconsistent results. ${ }^{31-37}$ The availability of potent, ritonavir-boosted PIs with better safety profiles has renewed interest in induction-maintenance approaches. The potential advantages of such ART simplification strategy include low pill burden, once-daily dosing, preservation of future options, avoidance of NRTI-based toxicity and lower cost. However, disadvantages include the possibility of lower potency, increased likelihood of treatment failure, especially with missed doses, lack of penetration into sanctuary sites (central nervous system, genital secretions), and the possible need for more frequent virologic monitoring. ${ }^{2}$

Until recently most of the trials of PI/r monotherapy have studied LPV/r. In a systematic review of 22 studies (including 6 randomized controlled trials) assessing the efficacy of PI/r monotherapy demonstrated slightly inferior efficacy of LPV/r monotherapy compared to standard treatment with LPV/r plus two NRTIs. ${ }^{38}$ One of these studies, the OK trial, evaluated maintenance with LPV/r monotherapy versus continuing LPV/r plus two NRTIs in HIV-infected patients with suppressed HIV replication. ${ }^{39,40}$ This was a randomized, controlled, open-label, multicenter, pilot trial. Adult patients were eligible if they had no history of virologic failure while receiving a PI, were receiving two NRTIs plus LPV/r (400/100 mg twice daily) for $>1$ month and had maintained an HIV RNA $<50$ copies/ $\mathrm{mL}$ for $>6$ months prior to enrollment. Forty-two patients were randomly assigned 1:1 to continue or stop the NRTIs. At baseline there were no significant differences between groups in median CD4 count (baseline or nadir), pre-ART viremia, or time with HIV RNA $<50$ copies $/ \mathrm{mL}$ prior to enrollment. After 48 weeks the percentage of patients maintaining virologic suppression by intention to treat (ITT) analysis was $81 \%$ for 
the monotherapy group versus $95 \%$ for the triple-therapy group $(P=0.34)$. Patients in whom monotherapy failed had significantly worse adherence than patients who remained virologically suppressed on monotherapy. Failure of monotherapy was not associated with emergence of PI resistance mutations, and patients were successfully re-suppressed with addition of pre-randomization NRTIs. Mean change in CD4 count was +70 cells $/ \mathrm{mm}^{3}$ (monotherapy) and +8 cells $/ \mathrm{mm}^{3}$ (triple therapy) $(P=0.27)$. Mean serum fasting lipids remained stable in both groups. No serious adverse events were observed. At 96 weeks LPV/r monotherapy - with reintroduction of NRTIs as needed - was noninferior to continuation of triple therapy. The incidence of adverse events leading to treatment discontinuation was significantly lower with monotherapy.

ACTG 5201 was a non-controlled pilot study $(\mathrm{n}=36)$ that evaluated simplified maintenance therapy with ATV/r alone in virologically suppressed patients. ${ }^{41,42}$ Thirty-four patients were included in the analysis of the primary end point after 24 weeks. 91\% did experience virologic success (absence of failure) through 24 weeks of simplified therapy. Resistance testing at failure did not identify PI resistance mutations. While promising, these data need confirmation in larger, randomized trials.

More recently, the $\mathrm{MONET}^{43}$ and $\mathrm{MONOI}^{44}$ trials provided data using DRV/r monotherapy. In MONET 256 patients with HIV RNA $<50$ copies/mL for over 24 weeks on current ART (NNRTI-based [43\%] or PI-based [57\%]), switched to DRV/r $800 / 100 \mathrm{mg}$ once daily, either as monotherapy $(\mathrm{n}=127)$ or with two NRTIs $(n=129)$. Treatment failure was defined as two consecutive HIV RNA levels above 50 copies $/ \mathrm{mL}$ by week 48, or switches off study treatment. DRV/r monotherapy was noninferior to continued triple therapy by time to loss of virologic response (TLOVR) analysis. ${ }^{43}$ MONOI was a prospective, open-label, non-inferiority, 96-week safety and efficacy trial in virologically suppressed patients on triple therapy who were randomized to a $\mathrm{DRV} / \mathrm{r}$ triple drug regimen or DRV/r monotherapy $(\mathrm{n}=225) .{ }^{44} \mathrm{In}$ the per protocol analysis, treatment success was $99 \%$ on DRV/r triple drug versus $94 \%$ on DRV/r monotherapy; similar results were found in intent-to-treat analysis. Only 3 patients experienced virologic failure on DRV/monotherapy and none on triple therapy. No PI resistance emerged in patients with plasma viral load $>50 \mathrm{c} / \mathrm{mL}$. DRV $/ \mathrm{r}$ monotherapy exhibited an efficacy rate of over $85 \%$, with concordant results in the intent-to-treat and per protocol analyses with respect to the magnitude of difference between arms, but discordant conclusions with respect to the noninferiority margin. Of note, patients failing DRV/r monotherapy had no emergence of new
DRV resistance mutations. Despite these promising results, the use of maintenance PI/r monotherapy after induction with standard ART remains controversial and investigational due the lack of definitive data and concerns about the overall longterm likelihood of treatment failure, especially with missed doses, concern about penetration into sanctuary sites (central nervous system, genital secretions), ${ }^{41}$ and the possible need for more frequent virologic monitoring. ${ }^{38}$

\section{Drug substitution or switch strategies}

Since the early years of the HAART era, ART toxicity and tolerability have been identified as the principal causes of treatment substitutions, switches, and interruptions. ${ }^{39,40}$ In addition to short-term side effects (eg, GI intolerance, skin rash, CNS symptoms) and toxicity (eg, bone marrow suppression), which have been implicated as causes of poor regimen persistence and efficacy, ${ }^{14,45,46}$ adverse events such as lactic acidosis, hepatitis, pancreatitis and longer-term toxicities (eg, peripheral neuropathy, lipodystrophy) have been major drivers of changes to patients' ART regimens. Thymidine and adenosine analog NRTIs, such as ZDV, d4T and ddI, have been implicated as the cause of many of these toxicities, which have required treatment changes, ${ }^{14,17}$ and which may have been compounded by lower body weight, as recently observed in a resource-limited setting. ${ }^{15,17}$

\section{In-class switches}

In patients with moderate to severe lipoatrophy, significant increases in subcutaneous fat were observed over 104 weeks after switching from a thymidine analog to TDF or ABC ${ }^{47}$ Substitution of ZDV or d4T for TDF has led to partial reversal of lipoatrophy. ${ }^{47}$ Individuals receiving ZDV/3TC in the SWEET study who were randomized to switch to TDF/ FTC experienced significant improvement in lipoatrophy measured by limb fat on dual-energy X-ray absorptiometry (DEXA) scan at 48 weeks, and also reported fewer adverse events related to mitochondrial toxicity than individuals continuing ZDV/3TC. ${ }^{48}$ In addition, other reasons for in-class switches might include peripheral neuropathy (d4T to other NRTI $)^{49,50}$ or CNS side-effects (EFV to nevirapine [NVP] ${ }^{51}$ or etravirine [ETR]). ${ }^{52}$ In a trial reported by Waters and colleagues, switching from EFV to ETR led to a significant reduction in overall CNS adverse events such as insomnia, abnormal dreams and nervousness. Lack of improvement for some events suggests other causative factors. ${ }^{52}$ ARV-related gastrointestinal side effects can also be a reason for in-class switch (eg, $\mathrm{LPV} / \mathrm{r}$ to ATV/r or DRV/r, AZT to other NRTI). ${ }^{45,53,54}$ Finally, 
the SWAN study, in which there was a switch from a stable PI-based to an ATV-based regimen, demonstrated significant improvements in lipids after switch. ${ }^{55}$

\section{Out-of-class switches}

In treatment experienced patients on a stable suppressive enfuvirtide (ENF)-containing regimen, raltegravir (RAL) has been safely substituted for ENF. ${ }^{56,57}$ The SWITCHMRK-1 and -2 studies randomized 707 patients on a suppressive LPV/rbased regimen to switch from LPV/r to RAL or to remain on LPV/r, while continuing background therapy consisting of at least two NRTIs. The switch was associated with more virologic failure than continued therapy with LPV/r. ${ }^{58}$ In contrast, SPIRAL was a 48-week Spanish open label trial with similar study design but different results $(n=273)$. In patients with sustained virological suppression on $\mathrm{PI} / \mathrm{r}$-based therapy, switching from a PI/r to RAL demonstrated noninferior efficacy compared to continued $\mathrm{PI} / \mathrm{r}$-based therapy, with improvement in lipid profile at 48 weeks. ${ }^{59}$ Also, in the ODIS trial $(n=222),{ }^{60}$ suppressed patients (HIV RNA $<50$ copies $/ \mathrm{mL}$ for $>24$ weeks) were randomly assigned to RAL $800 \mathrm{mg}$ QD, $400 \mathrm{mg}$ BID, or $400 \mathrm{mg}$ BID for the first 3 months then $800 \mathrm{mg}$ QD. There were no significant differences when comparing RAL twice daily to once daily. The reason for the difference in outcome in these trials is unclear. It is possible that the other agents used in combination with RAL had greater activity in SPIRAL than they did in SWITCHMRK. It has also been suggested that the difference might be accounted for by the longer period of virologic suppression before switch in SPIRAL.

When considering treatment switches to improve tolerability and/or reduce toxicity, dosing frequency or pill burden, it is critical to consider the potential for untoward consequences related to virologic efficacy and sustained viral load suppression. Depending upon the activity of the other agents in a combination regimen, it may be ill-advised to switch from an antiretroviral medication with a high genetic barrier to a resistance to one with a lower resistance barrier (eg, from a PI/r to RAL or an NNRTI). In the setting of a background regimen with reduced antiviral activity, a switch to an agent with a low genetic barrier could result in viral rebound with the emergence of resistance. In contrast, switching to drugs with comparable or higher genetic barriers is generally felt to be safe, contingent upon the full activity of such agents.

\section{Simplified ART regimen, adherence, and health care cost}

In developed countries, ART simplification often increases cost, as shown in a US study demonstrating that better
ART adherence was associated with decreased health care utilization but increased total costs because of the higher cost of ART $(60 \% \text { of total costs })^{61}$ compared to other patient-related direct costs (hospitalization, laboratory, clinic visits, etc). The exception is PI/r monotherapy and intermittent therapy, neither of which is recommended. Cost has also influenced treatment recommendations. For example, in the UK it is now recommended that $\mathrm{ABC}$ be prescribed preferentially over TDF, and that $\mathrm{ATV} / \mathrm{r}$ is the preferred PI-based regimen in order to reduce costs to the National Health Service. ${ }^{62}$ In the US, with the growth of AIDS Drug Assistance Programs (ADAP) waiting lists and the impending availability of generic versions of preferred antiretroviral medications, there is potential for a paradoxical "de-simplification" of ART, with movement away from fixed-dose, lower pill burden regimens, to reduce the cost of ART. There may be a requirement from third-party payers to use generic, non-coformulated agents rather than newer agents or fixed-dose combinations. ${ }^{63}$

The situation in developing countries is somewhat different because of the availability of numerous generic coformulated products and name-brand drugs at lower negotiated prices. The generic coformulation of $\mathrm{d} 4 \mathrm{~T} / 3 \mathrm{TC} / \mathrm{NVP}$ is commonly used in sub-Saharan Africa, has been show to be highly effective, and costs about $\$ 20$ per patient per month. ${ }^{5,49}$ In such settings, single-tablet, fixed-dose combinations improve the reliability and security of supplies, reduce the number of pills, simplify the dosing regimen, are easier to take, enhance adherence, reduce the potential for inappropriate sharing of drugs, ensure that the correct dosage of each component is taken. ${ }^{64}$

Following the 2010 World Health Organization (WHO) ART recommendations, ${ }^{3} \mathrm{~d} 4 \mathrm{~T}$ has been progressively phased out as a prefered first-line regimen in resource-limited settings and replaced by TDF, which has a better toxicity profile, ${ }^{45,65,66}$ thus requiring fewer treatment switches compared to $\mathrm{AZT}^{45}$ or $\mathrm{d} 4 \mathrm{~T}$. ${ }^{65}$ A generic version ofTDF has been registered and is available in some parts of Africa such as South Africa, Zambia, Botswana and Namibia. Until recently, the availability of TDF in the public sector in South Africa has generally been limited by the high costs of the drug. ${ }^{67}$ However, following an agreement with the Clinton Foundation HIV/AIDS Initiative (CHAI), Matrix Laboratories, an Indian-based generics manufacturer, has committed to market TDF-based FDCs at prices that are competitive with South Africa's first line d4T-based regimens. TDF-based FDCs marketed through the CHAI agreement cost less than the most commonly used first line regimen of $\mathrm{d} 4 \mathrm{~T}+3 \mathrm{TC}+\mathrm{EFV}$. While the cost is still slightly higher than the cost of d4T + 3TC + NVP, less than $30 \%$ of patients are on this regimen. The cost per patient per month of first line-regimens in the public sector for 
$\mathrm{d} 4 \mathrm{~T}+3 \mathrm{TC}+\mathrm{EFV}$ is US\$22; for $\mathrm{d} 4 \mathrm{~T}+3 \mathrm{TC}+\mathrm{NVP}$ is US $\$ 11$. The costs of TDF-containing FDCs procured through CHAI are US $\$ 17.50$ for TDF/3TC/EFV and US $\$ 19.92$ for TDF/ FTC/EFV. ${ }^{67}$ The 2010 South African Department of Health ARVs guidelines recommends that all new HIV-infected adult patients eligible for treatment (including pregnant women) be started on TDC + either 3TC or FTC + either EFV or NVP. Those who are already on $\mathrm{d} 4 \mathrm{~T}$ and tolerating it should remain on that drug unless they are at risk for complications (high BMI, low hemoglobin, older female) ${ }^{68}$

If TDF-based FDCs are produced at sufficient volumes, they can be produced and marketed at prices that are competitive with d4T-based regimens. South Africa is the largest ARV provider in the world and would therefore meet the volumes required by generic companies to produce and market TDF-based FDCs at competitive prices. It has been shown by Rosen and colleagues that TDF at the price of US\$17 per month in South Africa could substantially save on $\mathrm{d} 4 \mathrm{~T}$ toxicity management and offset roughly $20 \%$ of the higher price of TDF. However, further decrease in TDF cost would be needed to make it highly cost-effective. ${ }^{69}$

\section{Conclusion}

High rates of sustained virologic suppression have been achieved with modern ART regimens, with increased attention on between-regimen differences (pill burden, dosing frequency, tolerability and toxicity) as principal determinants of enduring treatment success. Improved treatment adherence and regimen persistence have been observed with once-daily, low pill burden regimens, largely as a function of the improved tolerability and better toxicity profile of such regimens, many of which include co-formulated antiretroviral agents. Intermittent ART strategies are not recommended, and there is insufficient evidence to support induction-maintenance approaches. The role of regimen simplification using drugs with better toxicity profiles and lower pill burdens and issues related to quality of life, drug cost and cost-effectiveness have important implications not only for individual patients, but also for health care policy, guidelines and programmatic implementation and ART distribution globally.

\section{Acknowledgments}

Jean Nachega is supported by The United States National Institutes for Allergy and Infectious Disease (NIAID-NIH), Division of AIDS (DAIDS): K23AI 068582-01; The US President's Emergency Plan for AIDS Relief (PEPFAR) program through the Fogarty International Center (FIC)/ NIH and Health Research Administration (HRSA)
Grant Award: T84HA21652-01-00 for Medical Education Partnership Initiative (MEPI); The European Developing Countries Clinical Trial Partnership (EDCTP) Senior Fellowship Award: TA-08-40200-021 and the Wellcome Trust Southern Africa Consortium for Research Excellence (SACORE): WT087537MA. Michael Mugavero is supported by the NIH; P30AI27767, R24AI067039, K23MH082641, and R21AI087360. Michele Zeier receives partial support from PEPFAR program via the US Agency for International Development, (USAID) under Cooperative Agreement No. 674-A-00-08-00009-00. The views expressed in this publication do not necessarily reflect those of PEPFAR, USAID or WHO.

\section{Disclosure}

In the last 12 months, Dr Jean Nachega has received consulting fees or participated in scientific advisory boards for Gilead Sciences and/or Boehringer-Ingelheim and is a speaker bureau member for Glaxosmithkline and Gilead Sciences. Dr Michael Mugavero has received consulting fees for participation in scientific advisory boards from Bristol-Myers Squibb, Gilead Sciences and Merck \& Co as well as research support from Bristol-Myers Squibb, Tibotec, Pfizer, and Definicare. Dr Joel Gallant has received consulting fees or participated in scientific advisory boards, data safety monitoring boards, or endpoint review committees for Bristol-Myers Squibb, Gilead Sciences, Merck and Company, Tibotec Therapeutics, and ViiV Healthcare. Johns Hopkins University has received research funding from Gilead Sciences for research conducted by Dr Joel Gallant.

\section{References}

1. Panel on Antiretroviral Guidelines for Adults and Adolescents Guidelines for the use of antiretroviral agents in HIV-1-infected adults and adolescents. Department of Health and Human Services. January 10, 2011;1-166. Available from: http://www.aidsinfo.nih.gov/ContentFiles/ AdultandAdolescentGL.pdf. Accessed 2011 Feb 14.

2. McKinnon JE, Mellors JW, Swindells S. Simplification strategies to reduce antiretroviral drug exposure: progress and prospects. Antivir Ther. 2009;14(1):1-12.

3. World Health Organization. Antiretroviral Therapy For HIV Infection Adults And Adolescents: Recommendations for a public health approach. 2010 revision. Available from: http://whqlibdoc.who.int/ publications/2010/9789241599764_eng.pdf.

4. Henry K, Brundage R, Weller D, Akinsete O, Shet A. Comparison of generic zidovudine plus lamivudine (Cipla, Duovir) and the GlaxoSmithKline brand (Combivir) tablets. J Acquir Immune Defic Syndr. 2004;35(5):537.

5. Vezina H, Henry K, Ravindran GD, et al. A randomized crossover study to determine bioequivalence of generic and brand name nevirapine, zidovudine, and lamivudine in HIV-negative women in India. J Acquir Immune Defic Syndr 2006;41(2):131-136.

6. Parienti JJ, Bangsberg DR, Verdon R, Gardner EM. Better adherence with once-daily antiretroviral regimens: a meta-analysis. Clin Infect Dis. 2009;48(4):484-488. 
7. Maitland D, Jackson A, Osorio J, et al. Switch Study Team. Switching from twice-daily abacavir and lamivudine to the once-daily fixed-dose combination tablet of abacavir and lamivudine improves patient adherence and satisfaction with therapy. HIV Med. 2008;9(8):667-672.

8. Wright D, Rodriguez A, Godofsky E, et al. Efficacy and safety of 48 weeks of enfuvirtide $180 \mathrm{mg}$ once-daily dosing versus $90 \mathrm{mg}$ twicedaily dosing in HIV-infected patients. HIV Clin Trials. 2008;9(2):73-82.

9. Boyle BA, Jayaweera D, Witt MD, et al. Randomization to once-daily stavudine extended release/lamivudine/efavirenz versus a more frequent regimen improves adherence while maintaining viral suppression. HIV Clin Trials. 2008;9(3):164-176.

10. Zajdenverg R, Badal-Faesen, Andrade-Villanueva J, et al. Lopinavir/ ritonavir (LPV/r) tablets administered once-(QD) or twice-daily (BID) with NRTIs in antiretroviral-experienced HIV-1 Infected subjects: results of a 48-week randomized trial (Study M06-802). 5th IAS Conference on HIV pathogenesis, Treatment and Prevention. Cape Town, South Africa. 2009 Jul 19-22. Available from: http://www.ias2009. org/pag/Abstracts.aspx?AID=1249.

11. Bangsberg DR, Ragland K, Monk A, et al. A single tablet regimen is associated with higher adherence and viral suppression than multiple tablet regimens in HIV+ homeless and marginally housed people. AIDS. 2010;24(18):2835-2840.

12. DeJesus E, Pozniak A, Gallant J, et al. Improvement in Fasting Lipids but Minimal Recovery of Limb Fat Were Seen 96 Weeks After Switching from Lamivudine/Zidovudine Plus Efavirenz to Fixed-Dose Efavirenz/Emtricitabine/Tenofovir DF in HIV-Infected Patients. 49th Interscience Conference on Antimicrobial Agents and Chemotherapy (ICAAC) September 12-15, 2009 San Francisco, CA USA. Poster\# H-1572. Available from: http://www.hivandhepatitis.com/2009icr/icaac/ pdfs/2_DeJesus.pdf. Accessed July 10, 2011.

13. Airoldi M, Zaccarelli M, Bisi L, et al. One-pill once-a-day HAART: a simplification strategy that improves adherence and quality of life of HIV-infected subjects. Patient Prefer Adherence. 2010;4:115-125.

14. Bae JW, Guyer W, Grimm K, Altice FL. Medication persistence in the treatment of HIV infection: a review of the literature and implications for future clinical care and research. AIDS. 2011;25(3):279-290.

15. Willig JH, Abroms $\mathrm{S}$, Westfall $\mathrm{AO}$, et al. Increased regimen durability in the era of once-daily fixed-dose combination antiretroviral therapy. AIDS. 2008;22(15):1951-1960.

16. Gardner EM, Burman WJ, Maravi ME, Davidson AJ. Selective drug taking during combination antiretroviral therapy in an unselected clinic population. J Acquir Immune Defic Syndr. 2005;40(3):294-300.

17. Vo TT, Ledergerber B, Keiser O, et al. Durability and outcome of initial antiretroviral treatments received during $2000-2005$ by patients in the Swiss HIV Cohort Study. J Infect Dis. 2008;197(12):1685-1694.

18. Dybul M, Chun TW, Yoder C, et al. Short-cycle structured intermittent treatment of chronic HIV infection with highly active antiretroviral therapy: effects on virologic, immunologic, and toxicity parameters. Proc Natl Acad Sci U S A. 2001;98(26):15161-15166.

19. Dybul M, Nies-Kraske E, Dewar R, et al. A proof-of-concept study of short-cycle intermittent antiretroviral therapy with a once-daily regimen of didanosine, lamivudine, and efavirenz for the treatment of chronic HIV infection. J Infect Dis. 2004;189(11):1974-1982.

20. Cohen CJ, Colson AE, Sheble-Hall AG, McLaughlin KA, Morse GD. Pilot study of a novel short-cycle antiretroviral treatment interruption strategy: 48-week results of the five-days-on, two-days-off (FOTO) study. HIV Clin Trials. 2007;8(1):19-23.

21. Cohen C, Colson A, Pierone G, et al. 5th International AIDS Society Conference on HIV Pathogenesis, Treatment and Prevention. The FOTO study: the 48 week extension to assess durability of the strategy of taking efavirenz, tenofovir and emtricitabine Five days On, Two days Off (FOTO) each week in virologically suppressed patients, Cape Town, South Africa. 2009 Jul 19-22. Ab\#MOPEB063. Available from http:// www.ias2009.org/pag/Abstracts.aspx?AID=3046.

22. Danel C, Moh R, Minga A, et al. Trivacan ANRS 1269 trial group. CD4-guided structured antiretroviral treatment interruption strategy in HIV-infected adults in west Africa (Trivacan ANRS 1269 trial): a randomised trial. Lancet. 2006;367(9527):1981-1989.
23. Ananworanich J, Gayet-Ageron A, Le Braz M, et al; Staccato Study Group. Swiss HIV Cohort Study. CD4-guided scheduled treatment interruptions compared with continuous therapy for patients infected with HIV-1: results of the Staccato randomised trial. Lancet. 2006;368(9534):459-465.

24. El-Sadr WM, Lundgren JD, Neaton JD, et al. Strategies for Management of Antiretroviral Therapy (SMART) Study Group. CD4+ count-guided interruption of antiretroviral treatment. $N$ Engl J Med. 2006;355(22):2283-2296

25. El-Sadr WM, Grund B, Neuhaus J, et al. Risk for opportunistic disease and death after reinitiating continuous antiretroviral therapy in patients with HIV previously receiving episodic therapy: a randomized trial. Ann Intern Med. 2008;149(5):289-299.

26. Lundgren JD, Babiker A, El-Sadr W, et al. Inferior clinical outcome of the CD4+ cell count-guided antiretroviral treatment interruption strategy in the SMART study: role of CD4+ Cell counts and HIV RNA levels during follow-up. Strategies for Management of Antiretroviral Therapy (SMART) Study Group. J Infect Dis. 2008;197(8):1145-1155.

27. Emery S, Neuhaus JA, Phillips AN, et al. Major clinical outcomes in antiretroviral therapy (ART)-naive participants and in those not receiving ART at baseline in the SMART study. J Infect Dis. 2008;197(8): $1133-1144$

28. Kaufmann GR, Elzi L, Weber R, et al. Interruptions of cART limits CD4 T-cell recovery and increases the risk for opportunistic complications and death. AIDS. 2011;25(4):441-451.

29. Havlir DV, Marschner IA, Hirsch MS, et al. Maintenance antiretroviral therapies in HIV-infected subjects with undetectable plasma HIV RNA after triple-drug therapy. $N$ Engl J Med. 1998;339(18): 1261-1268.

30. Pialoux G, Raffi F, Brun-Vezinet F, et al. Trilège (Agence Nationale de Recherches sur le SIDA 072) Study Team. A randomized trial of three maintenance regimens given after three months of induction therapy with zidovudine, lamivudine, and indinavir in previously untreated HIV-1-infected patients. N Engl J Med. 1998;339(18):1269-1276.

31. Opravil M, Hirschel B, Lazzarin A, et al. A randomized trial of simplified maintenance therapy with abacavir, lamivudine, and zidovudine in human immunodeficiency virus infection. $J$ Infect Dis. 2002;185(9):1251-1260.

32. Katlama C, Fenske S, Gazzard B, et al. TRIZAL study: switching from successful HAART to Trizivir (abacavir-lamivudine-zidovudine combination tablet): 48 weeks efficacy, safety and adherence results. HIV Med. 2003;4(2):79-86.

33. Markowitz M, Hill-Zabala C, Lang J, et al. Induction with abacavir/ lamivudine/zidovudine plus efavirenz for 48 weeks followed by 48-week maintenance with abacavir/lamivudine/zidovudine alone in antiretroviral-naive HIV-1-infected patients. J Acquir Immune Defic Syndr. 2005;39(3):257-264.

34. Mallolas J, Pich J, Penaranda M, et al. Induction therapy with trizivir plus efavirenz or lopinavir/ritonavir followed by trizivir alone in naive HIV-1-infected adults. AIDS. 2008;22(3):377-384.

35. Opravil M, Baumann D, Chave JP, et al. Long-term efficacy after switch from protease inhibitor-containing highly active antiretroviral therapy to abacavir, lamivudine, and zidovudine. AIDS. 2004; 18(16):2213-2215.

36. Delfraissy JF, Flandre P, Delaugerre C, et al. Lopinavir/ritonavir monotherapy or plus zidovudine and lamivudine in antiretroviral-naive HIV-infected patients AIDS. 2008;22(3):385-393.

37. Wilkin TJ, McKinnon JE, DiRienzo AG, et al. Regimen simplification to atazanavir-ritonavir alone as maintenance antiretroviral therapy: final 48 -week clinical and virologic outcomes $J$ Infect Dis. 2009;199(6):866-871.

38. Bierman WF, van Agtmael MA, Nijhuis M, Danner SA, Boucher CA. HIV monotherapy with ritonavir-boosted protease inhibitors: a systematic review. AIDS. 2009;23(3):279-291.

39. Arribas JR, Pulido F, Delgado R, et al. Lopinavir/ritonavir as singledrug therapy for maintenance of HIV-1 viral suppression: 48-week results of a randomized, controlled, open-label, proof-of-concept pilot clinical trial (OK Study). J Acquir Immune Defic Syndr. 2005;40(3): 280-287. 
40. Arribas JR, Delgado R, Arranz A, et al; OK04 Study Group. Lopinavirritonavir monotherapy versus lopinavir-ritonavir and 2 nucleosides for maintenance therapy of HIV: 96-week analysis. J Acquir Immune Defic Syndr. 2009;51(2):147-152.

41. Swindells S, DiRienzo AG, Wilkin T, et al. Regimen simplification to atazanavir-ritonavir alone as maintenance antiretroviral therapy after sustained virologic suppression. JAMA. 2006;296(7):806-814.

42. Karlstrom O, Josephson F, Sonnerborg A. Early virologic rebound in a pilot trial of ritonavir-boosted atazanavir as maintenance monotherapy. J Acquir Immune Defic Syndr. 2007;44(4):417-422.

43. Arribas JR, Horban A, Gerstoft J, et al. The MONET trial: darunavir/ ritonavir with or without nucleoside analogues, for patients with HIV RNA below 50 copies/ml. AIDS. 2010;24(2):223-230.

44. Katlama C, Valentin MA, Algarte-Genin G, et al. Efficacy of darunavir/ ritonavir as single-drug maintenance therapy in patients with HIV-1 viral suppression: a randomized open-label noninferiority trial, MONOI-ANRS 136. AIDS. 2010;24(15):2365-2374.

45. Gallant JE, DeJesus E, Arribas JR, et al; Study 934 Group. Tenofovir DF, emtricitabine, and efavirenz vs zidovudine, lamivudine, and efavirenz for HIV. $N$ Engl J Med. 2006;354(3):251-260.

46. Sax PE, Tierney C, Collier AC, et al; AIDS Clinical Trials Group Study A5202 Team. Abacavir-lamivudine versus tenofovir-emtricitabine for initial HIV-1 therapy. $N$ Engl J Med. 2009;361(23):2230-2240.

47. Moyle GJ, Sabin CA, Cartledge J, et al; RAVE (Randomized Abacavir versus Viread Evaluation) Group UK. A randomized comparative trial of tenofovir DF or abacavir as replacement for a thymidine analogue in persons with lipoatrophy. AIDS. 2006;20(16):2043-2050.

48. Fisher M, Moyle GJ, Shahmanesh M, et al; SWEET (Simplification With Easier Emtricitabine Tenofovir) Group UK. A randomized comparative trial of continued zidovudine/lamivudine or replacement with tenofovir disoproxil fumarate/emtricitabine in efavirenz-treated HIV-1-infected individuals. J Acquir Immune Defic Syndr. 2009;51(5): $562-568$.

49. Laurent C, Bourgeois A, Mpoudi-Ngolé E, et al. Tolerability and effectiveness of first-line regimens combining nevirapine and lamivudine plus zidovudine or stavudine in Cameroon. AIDS Res Hum Retroviruses. 2008;24(3):393-399.

50. Hawkins C, Achenbach C, Fryda W, et al. Antiretroviral durability and tolerability in HIV-infected adults living in urban Kenya. J Acquir Immune Defic Syndr. 2007;45(3):304-310.

51. Mehta U, Maartens G. Is it safe to switch between efavirenz and nevirapine in the event of toxicity? Lancet Infect Dis. 2007;7(11): 733-738.

52. Waters L, Fisher M, Winston A, et al. A phase IV, double-blind, multicentre, randomized, placebo-controlled, pilot study to assess the feasibility of switching individuals receiving efavirenz with continuing central nervous system adverse events to etravirine. AIDS. 2011; 25(1):65-71

53. Mallolas J, Podzamczer D, Milinkovic A, et al; ATAZIP Study Group. Efficacy and safety of switching from boosted lopinavir to boosted atazanavir in patients with virological suppression receiving a LPV/rcontaining HAART: the ATAZIP study. J Acquir Immune Defic Syndr. 2009;51(1):29-36.

54. Mills AM, Nelson M, Jayaweera D, et al. Once-daily darunavir/ritonavir vs lopinavir/ritonavir in treatment-naive, HIV-1-infected patients: 96-week analysis. AIDS. 2009;23(13):1679-1688.
55. Gatell J, Salmon-Ceron D, Lazzarin A, et al; SWAN Study Group. Efficacy and safety of atazanavir-based highly active antiretroviral therapy in patients with virologic suppression switched from a stable, boosted or unboosted protease inhibitor treatment regimen: the SWAN Study (AI424-097) 48-week results. Clin Infect Dis. 2007;44(11):1484-1492.

56. Grant PM, Palmer S, Bendavid E, et al. Switch from enfuvirtide to raltegravir in virologically suppressed HIV-1 infected patients: effects on level of residual viremia and quality of life. Clin Virol. 2009;46(4): 305-308.

57. De Castro N, Braun J, Charreau I, et al; EASIER ANRS 138 study group. Switch from enfuvirtide to raltegravir in virologically suppressed multidrug-resistant HIV-1-infected patients: a randomized open-label trial. Clin Infect Dis. 2009;49(8):1259-1267.

58. Eron JJ, Young B, Cooper DA, et al. Switch to a raltegravir-based regimen versus continuation of a lopinavir-ritonavir-based regimen in stable HIV-infected patients with suppressed viraemia (SWITCHMRK 1 and 2): two multicentre, double-blind, randomised controlled trials. Lancet. 2010;375(9712):396-407.

59. Martínez E, Larrousse M, Llibre JM, et al; SPIRAL Study Group. Substitution of raltegravir for ritonavir-boosted protease inhibitors in HIVinfected patients: the SPIRAL study. AIDS. 2010;24(11):1697-1707.

60. Vispo E, Barreiro P, Maida I, et al. Simplification from protease inhibitors to once- or twice-daily raltegravir: the ODIS trial. HIV Clin Trials. 2010;11(4):197-204.

61. Gardner EM, Maravi ME, Rietmeijer C, Davidson AJ, Burman WJ. The association of adherence to antiretroviral therapy with healthcare utilization and costs for medical care. Appl Health Econ Health Policy. 2008;6(2-3):145-155

62. Mandalia S, Mandalia R, Lo G, et al; NPMS-HHC Steering Group. Rising population cost for treating people living with HIV in the UK, 1997-2013. PLoS One. 2010;5(12):e15677.

63. The ADAP Watch. April 2011. Available from: http://www.nastad.org/ Docs/Public/InFocus/201148_ADAP\%20Watch\%20update\%20-\%20 4.8.11.pdf. Accessed on 2011 May 13.

64. Nachega JB, Leisegang R, Bishai D, et al. Association of antiretroviral therapy adherence and health care costs. Ann Intern Med. 2010;152(1): $18-25$.

65. Gallant JE, Staszewski S, Pozniak et al. Efficacy and safety of tenofovir DF vs stavudine in combination therapy in antiretroviral-naïve patients: a 3-year randomized trial. JAMA. 2004;292(2):191-201.

66. Milinkovic A, Martinez E, Lopez S, et al. The impact of reducing stavudine dose versus switching to tenofovir on plasma lipids, body composition and mitochondrial function in HIV-infected patients. Antivir Ther. 2007;12(3):407-415.

67. Treatment Action Campaign. Tenofovir: an explanation of why we need TDF-dose combinations and the steps to bring them to the market in South Africa. Available from: http://www.tac.org.za/community/ node/2802; Accessed on 2011 May 13.

68. Department of Health, Republic of South Africa. The South African Antiretroviral Treatment Guidelines 2010. Available from: http:// www.doh.gov.za/docs/factsheets/guidelines/art.pdf. Accessed on 2001 May 13.

69. Rosen S, Long L, Fox M, Sanne I. Cost and cost-effectiveness of switching from stavudine to tenofovir in first-line antiretroviral therapy regimens in South Africa. J Acquir Immune Defic Syndr. 2008;48(3):334-344.
Patient Preference and Adherence

\section{Publish your work in this journal}

Patient Preference and Adherence is an international, peer-reviewed open access journal focusing on the growing importance of patient preference and adherence throughout the therapeutic continuum. Patient satisfaction, acceptability, quality of life, compliance, persistence and their role in developing new therapeutic modalities and compounds to

\section{Dovepress}

optimize clinical outcomes for existing disease states are major areas of interest. This journal has been accepted for indexing on PubMed Central. The manuscript management system is completely online and includes a very quick and fair peer-review system. Visit http://www.dovepress.com/ testimonials.php to read real quotes from published authors. 